\title{
The Correlations Between TNF System and Memories in Chronic Insomnia Disorder
}

\author{
Lan Xia ${ }^{1}$, Yi-Jun $\mathrm{Ge}^{2}$, Fang Wang ${ }^{3}$, Ping $\mathrm{Qu}^{1}$ and Gui-Hai Chen ${ }^{2,}$ \\ ${ }^{1}$ Department of Neurology, the Second Affiliated Hospital of Anhui Medical University, Hefei, 230601, China \\ ${ }^{2}$ Department of Neurology (Sleep Disorder), the Affiliated Chaohu Hospital of Anhui Medical University, Hefei \\ (Chaohu), 238000, China \\ ${ }^{3}$ Department of Neurology, the First Affiliated Hospital of Anhui Medical University, Hefei, 230022, China
}

\begin{abstract}
Objectives: To explore the changes of serum levels of tumor necrosis factors (TNF- $\alpha$ and TNF- $\beta$ ) and their soluble receptors (sTNF-R1 and sTNF-R2), and analyze their relationship between sleep quality and memory in the patients with chronic insomnia disorder (CID).

Methods: Forty-four CID patients and 39 normal controls were enrolled in this study. Pittsburgh Sleep Quality Index (PSQI) and Nine-Box Maze Test (9BMT) were used to assess the sleep quality and memory function, respectively. The serum levels of TNF- $\alpha$, TNF- $\beta$, sTNF-R1 and sTNF-R2 were examined by protein-chip.

Results: Compared to the controls, the CID patients had significantly higher error numbers of the spatial working and object recognition memories $(P S<0.05)$ in the 9BMT, with higher serum levels of TNF- $\alpha$ and TNF- $\beta(P S<0.001)$, and lower levels of sTNF-R1 and sTNF-R2 (Ps < 0.001). The partial correlation analysis showed that the serum TNF- $\alpha$ and TNF- $\beta$ positively but sTNF-R1 and sTNF-R2 negatively correlated with PSQI scores $(P S<0.001)$. In the CID patients, the TNF- $\alpha$ positively correlated with the errors in the spatial working and object recognition memories $(P \mathrm{~S}<0.05)$, whereas the TNF- $\beta$ only positively correlated with the errors in the spatial working memory $(P<0.05)$.
\end{abstract}

Conclusions: The CID patients had increased TNF- $\alpha$ and TNF- $\beta$ and decreased sTNF-R1 and sTNF-R2 in peripheral blood, with the former being linked to specific memory impairments.

Keywords: Chronic insomnia disorder, Tumor necrosis factor, Soluble receptor, Memory.

\section{INTRODUCTION}

Chronic insomnia disorder (CID) is a subjective experience of persistent sleep initiation, maintenance difficulties and/or early awakening, which can lead to some kinds of daytime functional impairments [1]. The current evidence suggests that patients with CID have not only dysfunctions of subjective learning and memory [2] but also selective impairments of objective memories [3-5]. Spatial memory is responsible for identifying, coding, storage and retrieval of spatial information about the arrangement of objects or specific routes $[6,7]$. It is a higher-degree cognitive function, and involved in declarative memory, procedural memory, working memory, reference memory, as well as other memory systems [8]. In humans, spatial memory is considered to be the earliest impaired memory form not only during the normal aging but also in some learning and memory loss diseases, such as Alzheimer's disease [9]. The Nine Box Maze Test have been used to assess the spatial, non-spatial (object), working (trial dependent),

"Address correspondence to this author at the Department of Neurology (Sleep Disorders), the Affiliated Chaohu Hospital of Anhui Medical University, Hefei (Chaohu), 238000, China; Tel: +86-551-82324252; Fax: +86-551-82324252; E-mail: doctorcgh@163.com reference (trial independent) memories, and recognition memory simultaneously [9-11]. It is sensitive to the deficits of visuo-spatial memory [9]. Consistently, our previous studies showed that the modified Nine Box Maze Test can detect mild-damaged spatail working memory (SWM) and object recognition memory (ORcM) $[5,12]$ or and object working memory (OWM) [12] in the CID patients or SWM, OWM and spatial reference memory (SRM) in the chronic tensiontype headache patients [13]. However, the mechanisms of objectively special-impairment memories in the CID patients have not been known well.

To some degree, there is a co-relationship between sleep and inflammation. Some studies have shown that tumor necrosis factors- $\alpha$ (TNF- $\alpha$ ) and interleukin $1 \beta$ (IL$1 \beta)$ are involved in physiological sleep regulation, especially increased slow wave sleep [14-16]. Studies show that non-rapid eye movement sleep can be increased by both injection of exogenous low doses and enhance of endogenous production in IL-1 $\beta$ or TNF-a, and be inhibited by their inhibitors. Both IL-1 $\beta$ and TNF-a have been linked to a variety of clinical conditions involving sleep disorders, such as sleep apnea [17]. However, there were only few studies on changes of inflammatory factors, such as increased IL- 
6 , in the CID patient with small samples [18, 19]. Given the TNFs being linked to sleep regulation and memory physiologically and pathologically depending on the concentrations, we hypothesized that the TNFs are related to the maintenance of insomnia and impaired memory under insomnia condition. The aim of this study is to investigate the changes of serum levels of TNFs and their soluble receptors, i.e., soluble TNF receptor (sTNF-R) 1 and 2 which can alleviate the biological effects of TNFs on the membrane receptors, and the correlations between these proteins and memories in the patients with CID.

\section{SUBJECTS AND METHODS}

\subsection{Subjects}

Forty-four patients with CID were recruited from the Clinic of Sleep Disorder at the Affiliated Chaohu Hospital of Anhui Medical University. The patients were diagnosed with the criteria of International Classification of Sleep Disorders (Third Edition) [2]. The inclusive criteria consisted of those as follows: 1) patients complain of sleep difficulty with impairment of daytime functions; 2) illness duration was longer than 6 months; 3) age was at 18 to 60 years old; 4) educational level was higher than junior high school; 5) Pittsburgh Sleep Quality Index (PSQI) score was higher than 7 ; 6) Hamilton Depression Scale 17 items (HAMD-17) total score was less than 17. The exclusive criteria included: 1) taking antidepressants, antipsychotics and hypnotics, etc. within two weeks; 2) noncooperation and cannot complete the test due to visual and hearing damage; 3 ) suffering from chronic inflammatory diseases, brain organic diseases, serious medical diseases and drugs abuse; 4) comorbidity with other mental disorders; 5) being in pregnancy and lactation period. 39 good sleepers, who consisted of the control group, were collected from the Center of Health Examination in the same hospital, with no sleep and mood complaints and corresponding medical history with scores of PSQI $<5$ and HAMD-17 $<7$. The study was approved by the Ethics Committee of the Affiliated Chaohu Hospital of Anhui Medical University, and all subjects signed informed consent before study onset.

\subsection{Methods}

\subsubsection{General Information Collection}

The age, illness duration and educated years were collected, and the severity of insomnia and overall cognitive function were assessed by PSQI and Montreal Cognitive Assessment, respectively. PSQI is a standard scale accessing sleep quality via recall of sleep behaviors in the past month, consisting of subjective sleep quality, sleep latency, sleeping duration, sleep efficiency, somnipathy, use of hypnotic drugs and diurnal dysfunction [20]. The total score is at 0 to 21 , and in China score $\geq 7$ has high diagnostic sensitivity and specificity in distinguishing patients with poor sleep from normal subjects [21]. The Chinese Beijing Version of Montreal Cognitive Assessment (MoCA-C) is a brief and useful screening tool for mild general cognitive impairment under different clinical settings, which had been employed in the nation-wide screening of cognitive function in China [22]. It is used to evaluate the global cognition function of subjects, including visuo-spatial and executive functions, naming, attention, language, abstraction, short-term memory and orientation [22]. The total score is at 0 to 30 , and overall scores $\geq 26$ is considered as normal cognitive function in China [22].

\subsubsection{Special Memory Test}

Nine Box Maze Test [9] was mildly modified to evaluate multiple aspects of memory $[5,12,13]$, i.e., SWM, OWM, SRM, object reference memory (ORM), and ORcM. In brief, the protocol could be described as follow. In a spacious and bright room, a picture was put on one inside-wall as a place cue. A $120-\mathrm{cm}$-diameter table was put in the center of room, and 9 identical opaque containers with height $9 \mathrm{~cm}$ and diameter $8 \mathrm{~cm}$ were equidistantly located along the table border. There are two part of this test, namely, training and testing phases. During the object-familiarization phase, the subject was shown 10 common objects (namely, button, key, coin, battery, watch, pencil sharpener, nail clipper, shears, scotch tape and clothespin), and requested to name each object. In the training phase without performance being recorded, the subject was shown 2 random containers with 2 random objects from the object-familiarization phase, and asked to remember the objects and containers housed them. Then, the subject was required to move around the table twice clockwise and counterclockwise in sequence. After circling the subject was required to recognize the objects from a photograph of the 10common objects and the corresponding containers. If correctly, the test would proceed to the next step. If an incorrect response was given, the subject should continue to pointing out the objects or / and containers until a correct response. In the testing phase, the 
subject was requested to remember the 2 objects and their positions in the training phase. They would not be moved, and be tested at the end of the test to form object and spatial reference memories. The wrong numbers was recorded as ORM and SRM errors. The subject was told to remember every 2 objects from the object-familiarization phase and their locations being put into which 2 containers every trial. The error numbers of changed location and changed object were respectively recorded as performances of SWM and OWM. After 4 trials, subject was requested to recognize the objects in the training phase from a photograph. The photo was contained corresponding similar objects that had been used in the test. The wrong numbers were recorded as ORcM. The more errors were, the worse the memory performed.

\subsubsection{Serum Levels of TNF System}

The elbow intravenous blood was collected at 7:00 A.M. 9:00 A.M., with fasting, avoiding strenuous activity and mental stimulation. The samples were standing for $30 \mathrm{~min}$, and then were centrifuged for 5 $\mathrm{min}$ at $3000 \mathrm{r} / \mathrm{min}$, and frozen at $-80^{\circ} \mathrm{C}$. The serum levels of TNF- $\alpha$, TNF- $\beta$, sTNF-R1, and sTNF-R2 were tested by Ray Biotech Chip Detection Test according to the kit operation procedure.

\subsection{Statistical Analysis}

Statistical analysis was performed using SPSS16.0 package. First, the data were normalized. Normally distributional data were expressed with mean \pm standard deviation, and inter-group comparisons were performed using t-tests. Non-normally distributional data were expressed with P50 (P25, P75). Differences between groups were tested using a group-designed rank sum test (Mann Whitney U). The Spearman's and partial correlation amylases were used to explore the correlations between the serum levels of proteins and the error number in memory tests. Test level is $\alpha=$ 0.05 , both sides test.

\section{RESULTS}

\subsection{Background Data}

There were no statistics differences in age $(Z=$ $0.023, P=0.982)$, gender $(x 2=1.338, P=0.247)$, educated level $(Z=0.234, P=0.815)$ and MoCA-C score $(Z=0.650, P=0.516)$ between the CID group and the control group. The PSQI score in the CID group was significantly higher than that in the controls $(Z=7.864, P<0.001)$, see Table 1 .

\subsection{Memories and the Serum Levels of TNFs and Their Soluble Receptors}

The error numbers in the SWM $(Z=5.362, P<$ $0.001)$ and ORcM $(Z=3.260, P=0.001)$ in the CID group were significantly higher than those in the control group (see Table 2). There were no statistical differences in the error numbers in the ORM ( $Z=$ $0.310, P=0.756)$, SRM ( $Z=0.909, P=0.363)$ and OWM $(Z=1.741, P=0.082)$ between the two groups (see Table 2 and Figure 1).

The serum levels of TNF- $\alpha(t=8.402, P<0.001)$ and TNF- $\beta(Z=7.350, P<0.001)$ in the CID group were significantly higher, and the serum levels of sTNF-R1 $(Z=7.801, P<0.001)$ and sTNF-R2 $(Z=$ 4.526, $P<0.001$ ) were significantly lower than those in the control group (see Table 2 and Figure 2).

Table 1: The Background Data of Participants

\begin{tabular}{|c|c|c|}
\hline Groups & CID & Controls \\
\hline \hline $\mathrm{n}$ & 44 & $42.0(29.0,49.0)$ \\
\hline Age $(\mathrm{yr})$ & $40.5(29.5,52.8)$ & $20 / 19$ \\
\hline Male/ Female & $17 / 27$ & $15.0(10.0,16.0)$ \\
\hline Education (yr) & $12.0(9.5,16.0)$ & $27.0(26.0,28.0)$ \\
\hline MoCA-C (score) & $27.0(25.0,29.0)$ & $2.0(1.0,4.0)$ \\
\hline PSQI (score) & $16.0(15.0,18.0)^{*}$ & $0.00(0.00,2.00)$ \\
\hline HAMD-17 (score) & $9.54 \pm 3.38^{*}$ & \\
\hline
\end{tabular}

Abbreviations: CID, Chronic insomnia disorder; MoCA-C, Chinese-Beijing Version of Montreal Cognitive Assessment; PSQI, Pittsburgh Sleep Quality Index Expressions: Mean \pm SD (normally distributed variables) or P50 [P25, P75] (non-normally distributed variables). 
Table 2: Performance of Memories (Error Numbers) and Serum TNFs/ sTNF-Rs Levels

\begin{tabular}{|c|c|c|}
\hline Groups & CID $(\mathbf{n}=\mathbf{4 4 )}$ & Controls $(\mathbf{n}=39)$ \\
\hline \hline ORM & $0.0(0.0,1.0)$ & $0.0(0.0,1.0)$ \\
\hline SRM & $0.0(0.0,1.0)$ & $0.0(0.0,1.0)$ \\
\hline OWM & $0.0(0.0,2.0)$ & $1.0(1.0,2.0)$ \\
\hline SWM & $0.0(2.0,5.0)^{*}$ & $0.0(0.0,0.0)$ \\
\hline ORcM & $0.0(0.0,1.0)^{*}$ & $291.7 \pm 91.0$ \\
\hline TNF- $\alpha(\mathrm{pg} / \mathrm{mL})$ & $497.8 \pm 127.0^{*}$ & $141.4(118.6,176.6)$ \\
\hline TNF- $\beta(\mathrm{pg} / \mathrm{mL})$ & $215.8(201.8,241.9)^{*}$ & $231.3(179.3,358.8)$ \\
\hline sTNF-R1 $(\mathrm{pg} / \mathrm{mL})$ & $93.4 \pm 18.5^{*}$ & $27.3(21.5,38.0)$ \\
\hline STNF-R2 $(\mathrm{pg} / \mathrm{mL})$ & $19.05 \pm 6.10^{*}$ & \\
\hline
\end{tabular}

Abbreviations: CID, Chronic insomnia disorder; ORcM, object recognition memory; ORM, object reference memory; OWM, object working memory; PSQI, Pittsburgh Sleep Quality Index; SRM, spatial reference memory; sTNF-R, soluble tumor necrosis factor receptor; SWM, spatial working memory; TNF, tumor necrosis factor. Expressions: Mean \pm SD (normally distributed variables) or P50 [P25, P75] (non-normally distributed variables).

${ }^{*}$ Compared to the control group, $p<0.05$.

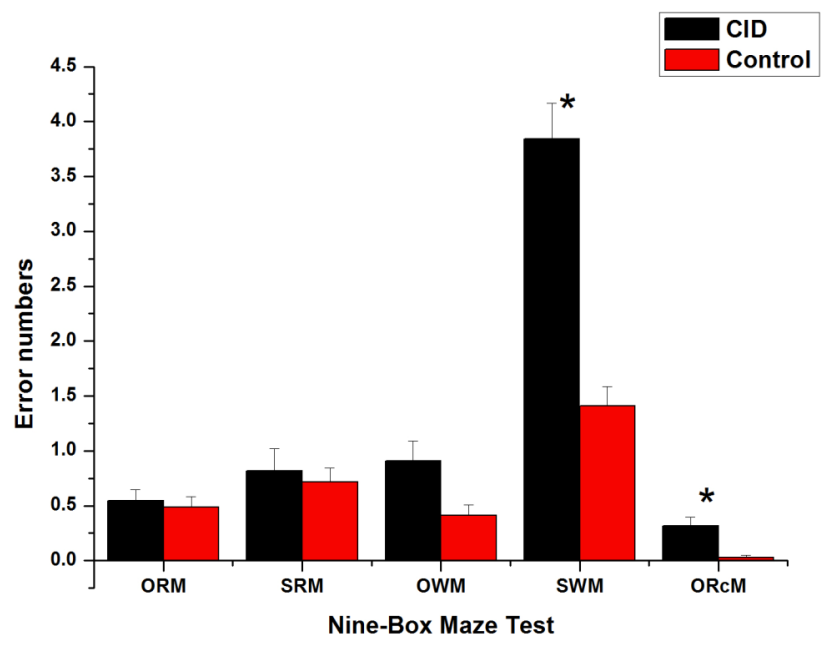

Figure 1: Performance of memories (error numbers) in the Nine-Box Maze Test. The patients with chronic insomnia disorder (CID) had more numbers in the spatial working memory (SWM) and object recognition memory (ORcM), not in the spatial reference memory (SRM), object working memory (OWM) and object reference memory (ORM), than the control subjects. ${ }^{*}$ Compared to the control group, $p<$ 0.05 .

\subsection{The Correlations between Sleep Quality and Serum TNFs and Soluble Receptors}

After controlling age, sex, educated year and HAMD-17 score, the partial correlation analysis showed that the serum levels of TNF- $\alpha$ and TNF- $\beta$ positively $(r=0.647$ and $0.757, P s<0.001)$, whereas the sTNF-R1 and sTNF-R2 negatively, correlated with the PSQI scores in all subjects $(r=-0.787$ and $-0.457, P$ S $<0.001$ ) (see Table 3).

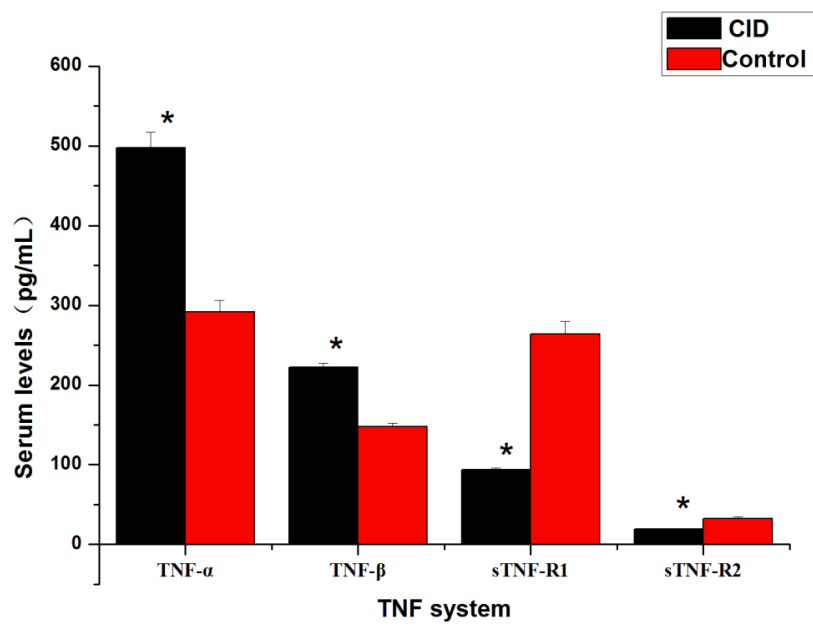

Figure 2: Serum TNFs/ sTNF-Rs levels. The patients with chronic insomnia disorder (CID) had higher tumor necrosis factor (TNF)- $\alpha$ and TNF- $\beta$, and lower soluble tumor necrosis factor receptor (sTNF-R)1 and STNF-R2, than the control subjects. ${ }^{*}$ Compared to the control group, $p<0.05$.

\subsection{Correlations between Serum Levels of TNFs System and Memory Performance}

In all subjects, after controlling age, sex, educated year and HAMD-17 scores, the partial correlation analysis showed that PSQI score was associated with serum levels of TNFs and its soluble receptors. Therefore, we explored the correlation between serum levels of TNFs and its receptors and memory performance adding PSQI score as a confounding factor to be controlled. The results showed that serum TNF- $\alpha$ level positively correlated with the error 
Table 3: The Correlations between Serum TNFs/ sTNF-Rs Levels and Memories $[r(p)]$

\begin{tabular}{|c|c|c|c|c|c|}
\hline \multirow{2}{*}{ Terms } & Measures & TNF- $\alpha$ & TNF- $\beta$ & sTNF-R1 & sTNF-R2 \\
\hline \hline \multirow{3}{*}{ PSQI score ${ }^{a}$} & & $0.647(0.000)^{*}$ & $0.757(0.000)^{*}$ & $-0.787(0.000)^{*}$ & $-0.457(0.000)^{*}$ \\
\hline \multirow{3}{*}{ Numbers of memory errors ${ }^{b}$} & ORM & $-0.065(0.569)$ & $0.005(0.962)$ & $-0.023(0.841)$ & $0.062(0.580)$ \\
\cline { 2 - 6 } & SRM & $-0.016(0.892)$ & $0.054(0.636)$ & $0.056(0.624)$ & $0.017(0.880)$ \\
\cline { 2 - 6 } & OWM & $-0.002(0.984)$ & $-0.184(0.107)$ & $-0.021(0.853)$ & $0.155(0.175)$ \\
\cline { 2 - 6 } & SWM & $0.266(0.019)^{*}$ & $0.279(0.013)^{*}$ & $0.140(0.220)$ & $0.136(0.233)$ \\
\hline \multirow{3}{*}{ Numbers of memory errors } & ORcM & $0.329(0.003)^{*}$ & $0.041(0.724)$ & $-0.123(0.283)$ & $-0.029(0.798)$ \\
\cline { 2 - 6 } & ORM & $-0.108(0.513)$ & $0.164(0.319)$ & $-0.224(0.170)$ & $0.040(0.817)$ \\
\cline { 2 - 6 } & SRM & $0.037(0.823)$ & $0.082(0.621)$ & $-0.153(0.353)$ & $0.204(0.213)$ \\
\cline { 2 - 6 } & OWM & $0.148(0.370)$ & $-0.295(0.068)$ & $-0.215(0.188)$ & $0.305(0.059)$ \\
\cline { 2 - 6 } & SWM & $0.380(0.017)^{*}$ & $0.414(0.009)^{*}$ & $-0.168(0.306)$ & $0.066(0.689)$ \\
\hline
\end{tabular}

Abbreviations: CID, Chronic insomnia disorder; ORcM, object recognition memory; ORM, object reference memory; OWM, object working memory; PSQI, Pittsburgh Sleep Quality Index; SRM, spatial reference memory; sTNF-R, soluble tumor necrosis factor receptor; SWM, spatial working memory; TNF, tumor necrosis factor

${ }^{a}$ Partial correlation analysis in all participants, controlling of age, sex, educated year and HAMD-17 score;

${ }^{\mathrm{b}} \mathrm{Partial}$ correlation analysis in all participants, controlling of age, sex, educated year, HAMD-17 and PSQI score;

${ }^{\mathrm{C}} \mathrm{Partial}$ correlation analysis in CID participants, controlling of age, sex, educated year, HAMD-17 and PSQI Score;

${ }^{*} p<0.05$.

numbers in the SWM $(r=0.266, P=0.019)$ and ORcM $(r=0.329, P=0.003)$, and the serum TNF- $\beta$ level only positively associated with the SWM errors. After separating the subjects, the results showed that in the CID group, the serum TNF- $\alpha$ level positively correlated with the error numbers in the SWM $(r=0.380, P=$ $0.017)$ and ORcM $(r=0.349, P=0.030)$, and serum TNF- $\beta$ level positively correlated with the error number in the SWM $(r=0.414, P=0.009)$. There were no significant correlations between serum levels of sTNFR1, sTNF-R2 and all tested types of memories ( $P S$ > 0.05) (see Table 3).

\section{DISCUSSIONS}

In the current study, we examined the serum levels of TNF system (including TNF- $\alpha$, TNF- $\beta$, sTNF-R 1 and sTNF-R2) and tested the SWM, OWM, SRM, ORM and ORcM with the 9BMT in all participates. Further, we analyzed their relationship between sleep quality and memory in the patients with CID. Compared to the controls, we found that: 1) the CID patients had significantly higher error numbers of the spatial working and object recognition memories in the 9BMT;2) the CID patients had higher serum levels of TNF- $\alpha$ and TNF- $\beta$, and lower levels of sTNF-R1 and sTNF-R2. Besides, the further correlation analyses showed that:
1) in all participants controlling the confounding factors the serum TNF- $\alpha$ and TNF- $\beta$ positively but sTNF-R1 and sTNF-R2 negatively correlated with PSQI scores; 2 ) in the CID patients, the TNF- $\alpha$ positively correlated with the errors in the SWM and ORCM, whereas the TNF- $\beta$ only positively correlated with the errors in the SWM.

Increasing findings have shown that CID patients possess significantly objective memory impairment $[3-5,23,24]$. Our current study once again demonstrated that CID patients had SWM and ORcM impairments (see Table 2), consistent with our previous findings [5, 12]. Nevertheless, little is known about the mechanism of memory impairment in the CID patients.

TNFs are a kind of cytokines produced by activated monocyte-macrophages with multi-functions, such as killing tumor cells, mediating inflammatory response, regulating neuronal activity (e.g., sleep regulation) [25]. The TNF- $\alpha$ and TNF- $\beta$, two main numbers of TNF superfamily derived from different-type cells, bind to the common receptors (TNF-R1 and TNF-R2) with similar biological functions. Studies have demonstrated that TNF- $\alpha$ can make specific effects on sleep-wake behavior [23]. Non-pyrogenic doses of TNF-a increased non-rapid eye movement sleep [26], but at 
high doses it inhibited non-rapid eye movement sleep and rapid eye movement sleep [16]. Blocking the effect of TNF- $\alpha$ can decrease the sleep pressure (sleep homeostasis) after sleep deprivation [16]. It has been shown that plasma TNF- $\alpha$ level in human is correlated with $\delta$ intensity in electroencephalogram [27].

There are a lot of studies on the relationships between sleep disorders and inflammatory factors. Many studies have shown that elevated $\mathrm{IL}-1 \beta$ and TNF- $\alpha$ are involved in sleepiness [28]. Similarly, enhanced secretion of TNF- $\alpha$ and IL- 6 are found in many conditions of sleep disorders, such as obstructive sleep apnea, chronic insomnia, narcolepsy and alcohol-related sleep disturbance [29]. A recent metaanalysis revealed that there was a link between sleep disturbances and two markers of systemic inflammation (i.e. C-reactive protein and IL-6) [30]. But there may be no correlation between sleep disturbance and TNF- $\alpha$ level because of low degree of statistical power [23]. The current study, at the first time, showed that the serum levels of both TNF- $\alpha$ and TNF- $\beta$ significantly increased in the CID patients, i.e., the levels of TNF- $\alpha$ and TNF- $\beta$ in the CID patients respectively increased by $70 \%$ and $50 \%$ compared to the control group (Table 2). Interestingly, our results also showed that the increased TNF- $\alpha$ level was associated with impairments of SWM and ORcM, and the increased TNF- $\beta$ level was also correlated with the SWM impairment (Table 3). These suggested that both TNF$\alpha$ and TNF- $\beta$, especially TNF- $\alpha$, may be associated with selective impairment of memory system, especially SWM, in the CID patients. This is consistent with the view-point that TNF- $\beta$ is more protective of nerve system than TNF- $\alpha$ [31].

STNF-Rs are natural inhibitors and modulators of TNFs activity [32]. There are two sTNF-Rs, i.e. sTNFR1 (p55) and sTNFR2 (p75). In many clinical situations, increased STNF-Rs levels are maker of disease activity [32]. Intracerebroventricular injection of sTNF-R1 impairs physiological sleep, suggesting that sTNF-R1 may be involved in the inhibition of sleep induced by TNFs [32]. Even the picogram-changes of sTNF-Rs level in the peripheral circulation may affect complex brain functions such as sleep and mood. There is one study explored the variation of STNF-R1 on sleep-wakefulness, and found that sTNF-R1 increased after 88-h whole sleep deprivation, but this effect was not seen in individuals who snapped 2-h/12$h$ [33]. A rhythmic study find that the 24-h variations of
sTNF-R1 and sTNF-R2 are characterized by a single cosine curve, and the apex time is near at 6 am (before waking up) [33]. The current study appeared to be the first time to describe the changed serum levels of sTNF-R1 and STNF-R2 in the CID patients, and the correlations between the sTNF-R1 and STNF-R2 levels and memories impairments. The results showed that the serum levels of these soluble receptors in the CID patients were significantly lower than those in the controls, i.e., sTNF-R1 was only about $40 \%$, and sTNFR2 was only about $70 \%$ of the control group (Table 2). However, there were no significant correlation between serum sTNF-R1 and sTNF-R2 levels and memory performance in the CID patients after controlling the factors of age, sex, educated years, HAMD-17 and PSQI scores (Tables 3). These results indicated that sTNF-R1 and sTNF-R2 might not be involved in the changes of the memory function in the CID patients directly.

In brief, the current study investigated the changes of objective memory function, serum levels of TNFs and their soluble receptors in the CID patients. The results suggested that the increased TNFs, rather than sTNF-Rs, levels in the CID patients may be related to their objective impairment of memories. This might provide a theoretical basis for neurobiological mechanisms of memory impairment in the CID patients. However, there are some limitations of this study. Firstly, to some degree, the sample size of this study was small and the sleep monitoring index was simple. Secondly, the certain memory aspects were chosen to examine here but not all memories. Thirdly, the levels of other inflammatory factors were not detected either. As a result, it is need to detect more factors to explore the co-relationship between inflammatory factors and memory impairment in more CID patients with more detailed history of illness.

\section{ACKNOWLEDGMENTS}

This paper was financially supported by Key Projects of Natural Science Research Projects for Universities in Anhui Province (No.KJ2016A322), Annal Plan in Key Science and Technology of Anhui Province (No. 1301043041), and Research Fund Project of Anhui Medical University (No. 2018xkj029).

\section{REFERENCES}

[1] Chen GH, Huang LQ, Zhao ZX. Insomnia. Sleep medicine, first Edition. Beijing: People's Medical Publishing House; 2016. 
[2] AASM. International classification of sleep disorders (3rd ed). American Academy of Sleep Medicine. Darien, IL: American Academy of Sleep Medicine; 2014.

[3] Shekleton JA, Flynn-Evans EE, Miller B, Epstein LJ, Kirsch $D$, Brogna LA, et al. Neurobehavioral performance impairment in insomnia: relationships with self-reported sleep and daytime functioning. Sleep 2014; 37(1): 107-116.

https://doi.org/10.5665/sleep.3318

[4] Fortier-Brochu E, Morin CM. Cognitive impairment in individuals with insomnia: clinical significance and correlates. Sleep 2014; 37(11): 1787-1798.

https://doi.org/10.5665/sleep.4172

[5] Chen GH, Xia L, Wang F, Li XW, Jiao CA. Patients with chronic insomnia have selective impairments in memory that are modulated by cortisol. Psychophysiology 2016; 53(10): 1567-1576.

https://doi.org/10.1111/psyp.12700

[6] Sharma S, Rakoczy S, Brown-Borg H. Assessment of spatial memory in mice. Life Sci 2010; 87: 521- 536. https://doi.org/10.1016/j.lfs.2010.09.004

[7] Moscovitch M, Nadel L, Winocur G, Gilboa A, Rosenbaum RS. The cognitive neuroscience of remote episodic, semantic and spatial memory. Curr Opin Neurobiol 2006; 16: 179-190. https://doi.org/10.1016/j.conb.2006.03.013

[8] Paul CM, Magda G, Abel S. Spatial memory: Theoretical basis and comparative review on experimental methods in rodents. Behav Brain Res 2009; 203: 151-164.

https://doi.org/10.1016/j.bbr.2009.05.022

[9] Abrahams S, Pickering A, Polkey CE, Morris RG. Spatial memory deficits in patients with unilateral damage to the right hippocampal formation. Neuropsychologia 1997; 35: 11- 24. https://doi.org/10.1016/S0028-3932(96)00051-6

[10] Pentland LM, Anderson VA, Dye S, Wood SJ. The Nine Box Maze Test: A measure of spatial memory development in children. Brain Cogn 2003; 52: 144- 154.

https://doi.org/10.1016/S0278-2626(03)00079-4

[11] Rahman Q, Abrahams S, Jussab F. Sex differences in a human analogue of the Radial Arm Maze: the "17-Box Maze Test". Brain Cogn 2005; 58: 312-317. https://doi.org/10.1016/j.bandc.2005.03.001

[12] Zhang P, Tan CW, Chen GH, Ge YJ, Xu J, Xia L, et al. Patients with chronic insomnia disorder have increased serum levels of neurofilaments, neuron-specific enolase and S100B: Does organic brain damage exist? Sleep Med 2018; 48:163-171.

https://doi.org/10.1016/j.sleep.2017.12.012

[13] Qu P, Yu JX, Xia L, Chen GH. Cognitive performance and the alteration of neuroendocrine hormones in chronic tension-type headache. Pain Pract 2018; 18: 8-17.

https://doi.org/10.1111/papr.12574

[14] Lorton D, Lubahn CL, Estus C, Millar BA, Carter JL, Wood $\mathrm{CA}$, et al. Bidirectional communication between the brain and the immune system: implications for physiological sleep and disorders with disrupted sleep. Neuroimmunomodulation 2006; 13(5-6): 357-374.

https://doi.org/10.1159/000104864

[15] Clinton JM, Davis CJ, Zielinski MR, Jewett KA, Krueger JM. Biochemical regulation of sleeps and sleep biomarkers. J Clin Sleep Med 2011; 7(5 Suppl): S38-S42. https://doi.org/10.5664/JCSM.1360

[16] Kapsimalis F, Richardson G, Opp MR, Kryger M. Cytokines and normal sleep. Curr Opin Pulm Med 2005; 11(6): 481484.

https://doi.org/10.1097/01.mcp.0000183062.98665.6b

[17] JM Krueger, JA Majde, DM Rector. Cytokines in immune function and sleep regulation. Handb Clin Neurol 2011; 98: 229-240.

https://doi.org/10.1016/B978-0-444-52006-7.00015-0
[18] Vgontzas AN, Zoumakis M, Papanicolaou DA, Bixler EO, Prolo $\mathrm{P}$, Lin HM, et al. Chronic insomnia is associated with a shift of interleukin-6 and tumor necrosis factor secretion from nighttime to daytime. Metabolism. 2002; 51(7): 887-892. https://doi.org/10.1053/meta.2002.33357

[19] Burgos I, Richter L, Klein T, Fiebich B, Feige B, Lieb K, et al. Increased nocturnal interleukin-6 excretion in patients with primary insomnia: a pilot study. Brain Behav Immun 2006; 20(3): 246-253.

https://doi.org/10.1016/j.bbi.2005.06.007

[20] Buysse DJ, Reynolds CF III, Monk TH, Berman SR, Kupfer DJ. The Pittsburgh Sleep Quality Index: a new instrument for psychiatric practice and research. Psychiatry Res 1989; 28(2): 193-213.

https://doi.org/10.1016/0165-1781(89)90047-4

[21] Wang $X D$, Wang $X L$, Ma $H(E d)$. Rating scales of mental health (Revised ed., in Chinese). Beijing, China: Chinese. Mental Health Journal Publishern 1999: p. 375-378.

[22] Yu J, Li J, Huang X. The Beijing version of the Montreal Cognitive Assessment as a brief screening tool for mild cognitive impairment: a community-based study. BMC Psychiatry 2012; 12: 156.

https://doi.org/10.1186/1471-244X-12-156

[23] Jewett KA, Krueger JM. Humoral sleep regulation; interleukin-1 and tumor necrosis factor. Vitam Horm. 2012, 89: 241-257.

https://doi.org/10.1016/B978-0-12-394623-2.00013-5

[24] Wei QG, Chen GH, Xia L, Wang F, Li XW. The differences of memories and sleep parameters and their correlations in patients with insomnia disorder in different subtpes: a clinical study. Chin J Neurol 2015; 48(9): 763-767.

[25] Croft M, Siegel RM. Beyond TNF: TNF superfamily cytokines as targets for the treatment of rheumatic diseases. Nat Rev Rheumatol 2017; 13(4): 217-233.

https://doi.org/10.1038/nrrheum.2017.22

[26] Shoham S, Davenne D, Cady AB, Dinarello CA, Krueger JM. Recombinant tumor necrosis factor and interleukin 1 enhance slow-wave sleep. Am J Physiol 1987; 253(1 Pt 2): R142-R149.

https://doi.org/10.1152/ajpregu.1987.253.1.R142

[27] Kapsimalis F, Basta M, Varouchakis G, Gourgoulianis K Vgontzas A, Kryger M. Cytokines and pathological sleep. Sleep Med 2008; 9(6): 603-614. https://doi.org/10.1016/j.sleep.2007.08.019

[28] Rockstrom MD, Chen L, Taishi P, Nguyen JT, Gibbons CM, Veasey SC, et al. Tumor necrosis factor alpha in sleep regulation. Sleep Med Rev 2018, 40: 69-78. https://doi.org/10.1016/j.smrv.2017.10.005

[29] Mullington J, Korth C, Hermann DM, Orth A, Galanos C Holsboer $\mathrm{F}$, et al. Dose-dependent effects of endotoxin on human sleep. Am J Physiol Regul Integr Comp Physiol 2000; 278(4): R947-R955. https://doi.org/10.1152/ajpregu.2000.278.4.R947

[30] Irwin MR, Olmstead R, Carroll JE. Sleep disturbance, sleep duration, and inflammation: A systematic review and metaanalysis of cohort studies and experimental sleep deprivation. Biol Psychiatry 2016; 80(1): 40-52. https://doi.org/10.1016/j.biopsych.2015.05.014

[31] Pae CU. Potential role of lymphotoxin-alpha (tumor necrosis factor-beta) in the development of schizophrenia. Med Hypotheses 2007; 68(6): 1359-1362.

https://doi.org/10.1016/j.mehy.2006.10.023

[32] Watanabe E, Buchman TG, Hirasawa H, Zehnbauer BA. Association between lymphotoxin-alpha (tumor necrosis factor-beta) intron polymorphism and predisposition to severe sepsis is modified by gender and age. Crit Care Med 2010; 38(1): 181-193.

https://doi.org/10.1097/CCM.0b013e3181bc805d 
[33] Haack M, Pollmacher T, Mullington JM. Diurnal and sleepwake dependent variations of soluble TNF- $\alpha$ and IL-2 receptors in healthy volunteers. Brain Behav Immun 2004;
18(4): 361-367.

https://doi.org/10.1016/j.bbi.2003.12.009

Received on 14-01-2020

Accepted on 24-01-2020

Published on 01-02-2020

http://dx.doi.org/10.15379/2409-3564.2020.07.01.01

(C) 2020 Xia et al.; Licensee Cosmos Scholars Publishing House.

This is an open access article licensed under the terms of the Creative Commons Attribution Non-Commercial License

(http://creativecommons.org/licenses/by-nc/3.0/), which permits unrestricted, non-commercial use, distribution and reproduction in any medium, provided the work is properly cited. 